\title{
ACTIVIDADES 2016
}

44th Annual Meeting of the International Neuropsychology Association

3 al 6 de febrero

Boston, Estados Unidos

Society for Cross-Cultural Research

Conference 2016

17 al 20 de febrero

Portland, Estados Unidos

International Family Therapy Association's 24th World Family Therapy Conference

30 de marzo al 2 de abril

Kona, Hawaii

XV Congreso Nacional Psicología de la Actividad Física y del Deporte y I Encuentro Internacional Entrenamiento Mental en el Deporte

30 de marzo al 2 de abril

Valencia, España

2nd World Conference on Personality

31 de marzo al 4 de abril

Buzios, Brasil

Jornada Interinstitucional Regional "Otto Kernberg: trabajando con su teoría y clínica"

7 al 9 de abril

Montevideo, Uruguay

12th Conference of the European Academy of Occupational Health Psychology 11 al 13 de abril

Atenas, Grecia

XVIII Jornadas Nacionales de Patología Dual

14 al 16 de abril

Madrid, España

XLIII Congreso Nacional del Consejo Nacional para la Enseñanza e Investigación en Psicología "La psicología como ciencia y su relación con otras disciplinas"

27 al 29 de abril

Veracruz, México

23rd International Symposium about Current Issues and Controversies in Psichiatry

28 al 30 de abril

Barcelona, España

International Meeting of the Psychonomic Society 5 al 8 de mayo

Granada, España

$7^{\text {a }}$ Convención Internacional de Psicología "Hominis 2016" "La Psicología potenciando el bienestar humano"

9 al 13 de mayo

La Habana, Cuba

VII Convención Intercontinental de Psicología

9 al 16 de mayo

La Habana, Cuba
Annual International Conference of the Association of Psychology and Psychiatry for Adults and Children "Recent Advances in Neuropsychiatric, Psychological and Social Sciences"

17 al 20 de mayo

Atenas, Grecia

VII Congreso Latinoamericano de Psicología de la Salud ALAPSA

23 al 27 de mayo

Cali, Colombia

Conferencia Internacional de Psicología Aplicada ICAP

26 al 27 de mayo

Tokio, Japón

28 ${ }^{\text {a }}$ Convención Anual de la Association for Psychological Science APS

26 al 29 de mayo

Chicago, Estados Unidos

$15^{\text {th }}$ World Association for Infant Mental Health Conference

29 de mayo al 2 de junio

Praga, República Checa

International Meeting of Traumatic Stress in Brazil

4 al 5 de junio

San Pablo, Brasil

VIII Congreso Internacional de Psicología y Educación

15 al 17 de junio

Alicante, España

8th World Congress of Behavioral and Cognitive Therapies 2016

22 al 25 de junio

Melbourne, Australia

8th European Conference of Positive

Psychology ECPP8

25 de junio al 1 de julio

Angers, Francia

37th STAR CONFERENCE - Stress and Anxiety in a Changing Society

6 al 8 de Julio

Zagreb, Croacia

31st International Congress of Psychology

24 al 29 de julio

Yokohama, Japón

XVII Congreso Latinoamericano de Análisis, Modificación del Comportamiento y Terapia Cognitivo Conductual

28 al 30 de julio

Panamá, Panamá 